\title{
A!
}

This is an electronic reprint of the original article.

This reprint may differ from the original in pagination and typographic detail.

Koponen, Lari M.; Nieminen, Jaakko O.; Ilmoniemi, Risto J.

\section{Minimum-energy Coils for Transcranial Magnetic Stimulation: Application to Focal Stimulation}

Published in:

Brain Stimulation

DOI:

10.1016/j.brs.2014.10.002

Published: 01/01/2015

Document Version

Peer reviewed version

Please cite the original version:

Koponen, L. M., Nieminen, J. O., \& Ilmoniemi, R. J. (2015). Minimum-energy Coils for Transcranial Magnetic Stimulation: Application to Focal Stimulation. Brain Stimulation, 8(1), 124-134.

https://doi.org/10.1016/j.brs.2014.10.002

This material is protected by copyright and other intellectual property rights, and duplication or sale of all or part of any of the repository collections is not permitted, except that material may be duplicated by you for your research use or educational purposes in electronic or print form. You must obtain permission for any other use. Electronic or print copies may not be offered, whether for sale or otherwise to anyone who is not an authorised user. 


\title{
Minimum-energy coils for transcranial magnetic stimulation: application to focal stimulation
}

\author{
Lari M. Koponen, Jaakko O. Nieminen, and Risto J. Ilmoniemi
}

September 26, 2014

\begin{abstract}
Background: In transcranial magnetic stimulation (TMS), the stimulation-coil current is typically increased from 0 to over $5000 \mathrm{~A}$ in less than $100 \mathrm{\mu s}$. At the peak current, the energy stored in the magnetic field is over $300 \mathrm{~J}$. Thus, the average power during a pulse exceeds $3 \mathrm{MW}$; the stimulator needs to be built from high-power electronics. The power requirements often limit the duration and frequency of repetitive TMS, for example, via coil heating.

Objective: We introduce a method for finding the minimum-energy solution for a TMS coil with given focality constraints.

Methods: This optimization is performed by using a spherically symmetric head model and by expressing the coil as a continuous surface current density, which is eventually discretized to form the coil windings. For the optimization, we defined TMS focality separately for the directions parallel and perpendicular to the maximum induced electric field.

Results: The computational model used for optimization was verified by manufacturing a prototype coil and measuring the electric field it induces in a spherically symmetric conductor. The optimized coil design requires significantly less power than existing TMS coil designs (a 73\% reduction compared to an existing TMS coil with similar focality).

Conclusion: The described method allows for more-efficient, morefocal TMS coils, which may reduce coil heating and the coil click.
\end{abstract}




\section{Introduction}

TMS coil focality and energy efficiency are intimately coupled; more energy is needed for producing a more focal stimulus. In this paper, we introduce a method for designing transcranial magnetic stimulation (TMS) coils that minimize the energy required for a given-focality pulse and evaluate whether it is reasonable to make the induced electric field (E-field) more focal than that of present figure-of-eight coils. In order to reach this goal, we will first introduce an efficient way to calculate the induced electric field inside the head. This method is based on continuous surface currents, vector-valued spherical harmonics, and the spherically symmetric head model. As will be evident, this approach is suitable for finding minimum-energy coils for any desired stimulus.

The »industrial standard» for focal TMS is a hand-held figure-of-eight coil. With navigated TMS, the positional accuracy of the stimulus site for such a system has been estimated to be $6 \mathrm{~mm}$ root mean square [1]. This figure includes all error sources, such as imperfections in the subject-specific magnetic resonance image giving the geometry of the head and approximations in the induced Efield computation model. This positional uncertainty is significantly smaller than the diameter of the region of high E-field for typical TMS coils [2].

During the first quarter-century of TMS, several coil designs have been introduced. The most notable of these are: round coil (the first design to exist, inducing non-focal stimulation, Ref. [3]), figure-of-eight coil (the standard solution for focal stimulation, two circular coils with counter-circulating currents next to each other with a focal point between these two circular coils, Ref. [4]), slinky coil (where multiple circular coils are joined into a fan-like assembly above the target, induced electric field similar to that of a figure-of-eight coil but with reduced side maximums, Ref. [5]), four-leaf coil (two figure-of-eight coils combined, suitable for short-range focal stimulation, Ref. [6]), and Hesed coil (a complicated coil geometry including radial coil elements designed for deep brain stimulus, Ref. [7]). In this paper, we do not use prior knowledge on the optimal coil design, rather we let the minimum-energy condition determine the coil geometry.

There exist several methods for calculating the induced electric field of a TMS coil. These include methods that are based on the boundary element method (BEM) and the finite element method (FEM). These methods are essentially numerical solvers for the Maxwell's equations and offer almost no insight to the general properties of the induced electric field. However, they allow for accurate modeling of the head. In addition to this kind of methods, there are TMS-specific methods, like the one by Eaton [8], where the induced electric field of a coil is calculated as a line integral of the coil current using sphericalharmonic series. The method introduced in this paper gives the induced electric field as a weighted sum of coil current distribution components. This solution shows, for example, that for an arbitrary TMS coil the E-field pattern is a kind of a mirror image (with a minus sign) of the coil current distribution, with the higher spatial frequencies being attenuated faster as a function of depth than the lower spatial frequencies. 


\section{Computation of the induced E-field}

In this section, we derive an expression for the E-field induced by an arbitrary time-dependent spherical surface current density in the spherically symmetric head model [9]. To obtain this, we first derive an expression for the magnetic field $\boldsymbol{B}$ due to a surface current density; for coil optimization purposes, we will also evaluate the energy stored in such a field. Appendix A introduces the necessary mathematical tools for this section, including the magnetic scalar potential, $V$; the real spherical harmonics, $Y$; and the real vector spherical harmonics, $\boldsymbol{Y}$. Section 2.4 justifies using a single-spherical-current-layer model in solving the TMS-coil optimization problem.

\subsection{The magnetic field of a spherical current distribution}

Let us consider a divergence-free surface current density $\boldsymbol{K}(\theta, \phi)$ on a spherical shell with radius $R$. Because the real vector spherical harmonics of Eq. (A.10) form a complete orthogonal set for divergence-free vector fields on a spherical shell, the general spherical surface current distribution is of the form

$$
\boldsymbol{K}(\theta, \phi)=\sum_{l=1}^{\infty} \sum_{m=-l}^{l} i_{l m} \boldsymbol{Y}_{l, l}^{m}(\theta, \phi) / R,
$$

where the constants $i_{l m}$ define the amplitudes of the different current-density modes. In this case, the magnetic scalar potential from Eq. (A.6) is finite everywhere. Thus, inside the spherical shell, we have

$$
V_{\mathrm{int}}(r, \theta, \phi)=\sum_{l=0}^{\infty} \sum_{m=-l}^{l} a_{l m} r^{l} Y_{l}^{m}(\theta, \phi),
$$

and, on the outside,

$$
V_{\mathrm{ext}}(r, \theta, \phi)=\sum_{l=0}^{\infty} \sum_{m=-l}^{l} b_{l m} r^{-(l+1)} Y_{l}^{m}(\theta, \phi) .
$$

Using Eqs. (2), (A.3), and (A.11), we obtain the magnetic field inside the spherical shell:

$$
\begin{aligned}
\boldsymbol{B}_{\mathrm{int}}(r, \theta, \phi) & =-\mu_{0} \nabla V_{\mathrm{int}}=-\mu_{0} \sum_{l=0}^{\infty} \sum_{m=-l}^{l} a_{l m} \nabla\left[r^{l} Y_{l}^{m}(\theta, \phi)\right] \\
& =-\mu_{0} \sum_{l=0}^{\infty} \sum_{m=-l}^{l} a_{l m} \sqrt{l(2 l+1)} r^{l-1} \boldsymbol{Y}_{l, l-1}^{m}(\theta, \phi) .
\end{aligned}
$$

Similarly, Eqs. (2), (A.3), and (A.9) give us the magnetic field outside the spherical shell:

$$
\begin{aligned}
\boldsymbol{B}_{\mathrm{ext}}(r, \theta, \phi) & =-\mu_{0} \nabla V_{\mathrm{ext}}=-\mu_{0} \sum_{l=0}^{\infty} \sum_{m=-l}^{l} b_{l m} \nabla\left[r^{-(l+1)} Y_{l}^{m}(\theta, \phi)\right] \\
& =-\mu_{0} \sum_{l=0}^{\infty} \sum_{m=-l}^{l} b_{l m} \sqrt{(l+1)(2 l+1)} r^{-(l+2)} \boldsymbol{Y}_{l, l+1}^{m}(\theta, \phi) .
\end{aligned}
$$


Combining this with the boundary condition between the internal and external magnetic field [10],

$$
\boldsymbol{B}_{\text {ext }}(R, \theta, \phi)-\boldsymbol{B}_{\text {int }}(R, \theta, \phi)=\mu_{0} \boldsymbol{K}(\theta, \phi) \times \boldsymbol{e}_{r},
$$

we obtain the expression for the magnetic field:

$$
\boldsymbol{B}(r, \theta, \phi)=\mu_{0} \sum_{l=1}^{\infty} \sum_{m=-l}^{l} i_{l m}\left\{\begin{array}{ll}
(-1) \sqrt{\frac{l+1}{2 l+1}} \frac{r^{l-1}}{R^{l}} \boldsymbol{Y}_{l, l-1}^{m}(\theta, \phi), & r<R \\
\sqrt{\frac{l}{2 l+1}} \frac{R^{l+1}}{r^{l+2}} \boldsymbol{Y}_{l, l+1}^{m}(\theta, \phi), & r>R
\end{array} .\right.
$$

\subsection{The magnetic field energy of the spherical surface cur- rent distribution}

The energy stored in a magnetic field is

$$
U=\frac{1}{2 \mu_{0}} \int_{\text {all space }} \boldsymbol{B}^{2} d V .
$$

Substituting the expression for the magnetic field, Eq. (7), into this equation and applying the orthogonality of the spherical harmonics under angular integration (Eq. (A.12)) to remove the cross terms yields that the energy of the magnetic field is the sum of the energies of individual spherical-harmonic current components. This is consistent with the fact that mutual inductances are zero for all spherical-harmonic surface-current-density modes. By carrying out the radial integration, we obtain

$$
U=\frac{\mu_{0}}{2} \sum_{l=1}^{\infty} \sum_{m=-l}^{l} \frac{i_{l m}^{2} R}{2 l+1}
$$

\subsection{Induced electric field}

The electric field induced inside a spherically symmetric conductor by an external coil can be calculated using the triangle construction $[11,12]$. The construction exploits the fact that an external coil cannot induce radial currents into a spherically symmetric conductor. Thus, when a changing magnetic field induces an electromotive force (EMF) into a closed loop 012 (Fig. 1), the induced radial electric field is zero, and thus

$$
\oint_{\partial 012} \boldsymbol{E} \cdot d \boldsymbol{l}=\int_{1}^{2} \boldsymbol{E} \cdot d \boldsymbol{l} .
$$

The EMF can be calculated using the third Maxwell equation-Faraday's law of induction-

$$
\nabla \times \boldsymbol{E}=-\frac{\partial \boldsymbol{B}_{\mathrm{int}}}{\partial t},
$$

which equals

$$
\oint_{\partial 012} \boldsymbol{E} \cdot d \boldsymbol{l}=-\int_{012} \frac{\partial \boldsymbol{B}_{\text {int }}}{\partial t} \cdot d \boldsymbol{S} .
$$

Because $\boldsymbol{E}$ is a smooth function, we can solve its value as a function of position by calculating the EMF of a triangular loop of infinitesimal width and 


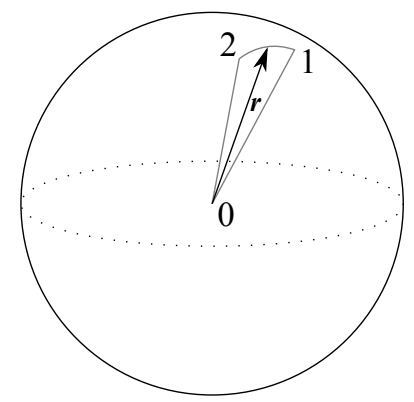

Figure 1: The triangle construction.

dividing this by the distance between points 1 and 2 . To get both the $\theta$ and the $\phi$ components (the $r$ component is zero), we have to do this twice for each location with two perpendicular triangular loops.

Performing the integrations at the limit of narrow triangles yields the expressions for the tangential components of $\boldsymbol{E}$. It turns out that the electric field due to each $(l, m)$ term in Eq. (1) can be expressed with just one real vector spherical harmonic function, and thus

$$
\boldsymbol{E}(r, \theta, \phi, t)=-\mu_{0} \sum_{l=1}^{\infty} \sum_{m=-l}^{l} \frac{\partial i_{l m}(t)}{\partial t} \frac{1}{2 l+1}\left(\frac{r}{R}\right)^{l} \boldsymbol{Y}_{l, l}^{m}(\theta, \phi)
$$

This equation shows that the induced field pattern is a kind of a mirror image (the minus sign in Eq. (13)) of the surface current density, with the higher spatial frequencies being attenuated faster as a function of depth than the lower ones.

\subsection{Justifying the single-spherical-current-layer model}

Until now, we have not fixed the radius of the surface current density distribution, $R$. From Eqs. (9) and (13) it is obvious that $R$ should be as small as possible for each spherical-harmonic component in order to achieve minimumenergy stimulation. This is an intuitive choice, as it both maximizes the induced electric field (Eq. (13)) and minimizes the energy (Eq. (9)) for a given current.

However, the intuition could easily be mistaken as the energy is proportional to the square of the current. Thus, it might actually be beneficial to move a small proportion of the current into a more distant spherical shell. Whether this is beneficial depends on the mutual inductances between these spherical shells.

For the spherical current shells, it can be shown with calculus of variations that it is not beneficial to split the current into smaller proportions (Appendix B). In Appendix B, we also show that radial currents do not introduce anything new to the induced electric field in the spherically symmetric head model but increase the energy stored in the field. This means that an arbitrary current distribution outside the head can be substituted with a tangential current distribution requiring less energy for the same induced current pattern. Thus, a single as-close-to-target-as-possible spherical current layer is a global optimum among the set of all current density distributions from the inductive energy point of view. 


\section{TMS-coil optimization method}

Eq. (13) gives the electric field at a point inside a spherically symmetric conductor enclosed inside the spherical current shell (i.e., at a point inside the head) for an arbitrary surface current density specified by Eq. (1). For an arbitrary current density with uniform pulse waveform, the electric field is

$$
\boldsymbol{E}(r, \theta, \phi)=-\mu_{0} \frac{\partial f(t)}{\partial t} \sum_{l=1}^{\infty} \sum_{m=-l}^{l} i_{l m} \frac{1}{2 l+1}\left(\frac{r}{R}\right)^{l} \boldsymbol{Y}_{l, l}^{m}(\theta, \phi)
$$

where $f(t)$ is the waveform of the current pulse and $i_{l m}$ its spatial amplitude spectrum. Combining this with the magnetic field energy from Eq. (9), we clearly see that the electric field of the higher-order terms is more expensive in terms of energy than that of the lower-order terms. Thus, truncating the spherical-harmonic series should not have a significant effect on minimum-energy solutions. ${ }^{1,2}$

\subsection{Defining the focality of a stimulus}

For now, we have considered a spherically symmetric head. Even though human head is not actually globally spherically symmetric it is nearly spherical locally. However, the symmetry does not hold for the sensitivity for stimulation. This is because the cortex of the brain is folded into gyri and sulci and the brain responds maximally to TMS when the induced electric field is perpendicular to a sulcus $[13,14]$.

If we want to have a focal stimulus, we must minimize the area where the induced electric field is strong. However, the direction perpendicular to the direction of the induced electric field is more important in defining focality than the direction parallel to the induced electric field. This is because the former induces stimulation right next to the target area; the latter affects the other side of the gyrus (for a stimulus perpendicular to a sulcus).

Previously, there have been multiple definitions for the focality. In Ref. [15], Thielscher and Kammer measured the focality of a stimulus based on the surface area at the target depth where the induced E-field exceeds a certain fraction of the maximum induced E-field. When the threshold value is $50 \%$, the focality of a typical figure-of-eight coil is $25-35 \mathrm{~cm}^{2}$ (assuming a cortical radius of $70 \mathrm{~mm}$ ). In Ref. [2], Deng et al. defined another measure for the focality, effective surface area,

$$
S_{1 / 2}=\frac{V_{1 / 2}}{d_{1 / 2}}
$$

where $V_{1 / 2}$ is the volume inside the brain where the stimulus is over $50 \%$ of the maximum and $d_{1 / 2}$ is the maximum depth where the stimulus is over $50 \%$ of the maximum. This metric takes into account that it is harder to have a focal stimulus deeper in the head.

However, using the $50 \%$ boundary is not always ideal. This is because of two things. First, for a typical stimulus strength, $50 \%$ of the stimulus maximum

\footnotetext{
${ }^{1}$ Obviously, the higher order terms include higher spatial frequencies than the lower-order terms. Some of these are needed for more focal stimuli.

${ }^{2}$ The second power in Eq. (9) makes it beneficial to have little of some high-order components. However, with a reasonable truncation cut-off point this difference is negligible.
} 
causes little activation of the cortex [16, 17]; second, for typical figure-of-eight coils the distance from the target to the $50 \%$ boundary is $2-5 \mathrm{~cm}$, much farther than the positional accuracy. Because of this, we propose the following definition for focality:

1. The focal region of a TMS stimulus is the area where the energy density of the stimulation exceeds $50 \%$ of the maximum. That is, the electric field is over $1 / \sqrt{2} \approx 70 \%$ of the maximum.

2. The focality is measured in two directions, one parallel to the direction of the maximum stimulus and one perpendicular to it. These measures are expressed with full-width at half-maximum (FWHM) values. The focal region is limited by the four great circles drawn at the four FWHM points perpendicular to their direction from the target point (Fig. 2).

This definition works well only for focal stimulus, that is, when there is a clear target with maximum stimulus.

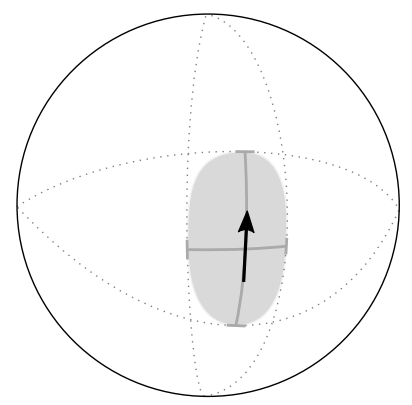

Figure 2: The focal region. The black arrow represents the induced electric field direction at the target, within the light gray area the induced electric field is over $1 / \sqrt{2}$ of the maximum, the solid gray lines define the parallel and perpendicular FWHM, and the dotted gray lines depict the great circles surrounding the focal region. Notice that the widest point does not need to be directly in either of the two FWHM directions.

\subsection{Optimization problem}

From Eqs. (9) and (14), we see that a focal pulse requires more energy than a less focal one. Thus, finding the surface current density with minimum energy for given constraints is an optimization problem. This problem is presented in Table 1. (We set $\partial f(t) / \partial t=1 /(100 \mu \mathrm{s})$ for a 100 - $\mu$ s-rise-time pulse.)

The problem in Table 1 include non-linear constraints for the norm of the induced electric field. Optimization problems with non-linear constraints are in general much harder to solve than ones with only linear constraints. In order to make the optimization more efficient, we can approximate the norm constraint (the endpoint of the E-field vector lies inside a circle with a given radius) with a few linear constraints (the endpoint lies inside a regular convex polygon). With 16 linear constraints, the norm can be approximated with maximum error of less than $1 \%$. Now, we have an optimization problem with a convex feasibility set and a convex objective function. This ensures that a local minimum is also the 
Table 1: Minimization problem. Subscript || refers to the component parallel with the target field and $\perp$ refers to the component perpendicular to it.

\begin{tabular}{lll}
\hline Optimization variables & $\boldsymbol{i}=\left\{i_{l m}\right\}$ & \\
Objective function & $U(\boldsymbol{i})$ & \\
Equality constraints & $E_{||, 0}=100 \mathrm{~V} / \mathrm{m}$ & at the focal point \\
& $E_{\perp, 0}=0$ & at the focal point \\
Inequality constraints & $|\boldsymbol{E}| \leq\left|\boldsymbol{E}_{0}\right|$ & everywhere \\
& $|\boldsymbol{E}| \leq\left|\boldsymbol{E}_{0}\right| / \sqrt{2}$ & outside the focal region
\end{tabular}

global minimum [18]. As the objective function is always positive, there exists a minimum if there exists a feasible point. ${ }^{3}$ Thus, the approximate solution for the global optima of the optimization problem can be found quickly with the interior point method.

\subsection{Transforming the optimization problem into linear al- gebra}

The spherical harmonics in Eq. 14 are expensive to calculate. For this reason, it is beneficial to calculate them only once for the optimization. This can be done with the following method.

- Take $N$ points where you want to compute the induced electric field from inside the sphere.

- Calculate $E_{\theta}(r, \theta, \phi)$ and $E_{\phi}(r, \theta, \phi)$ per unit current at each of these points for all $l \in\left\{1, \ldots, l_{\max }\right\}, m \in\{-l, \ldots, l\}$.

- Form an $\left[\left(l_{\max }\right)^{2}+2 l_{\max }\right] \times 2 N$ matrix $\mathbf{A}$ from these values.

- Enforce the constraints for the inner product of linear combinations of the rows of $\mathbf{A}$ and the vector $\boldsymbol{i}$. (For the 16-gon estimate of the norm, the final constraint matrix has the size of $\left[\left(l_{\max }\right)^{2}+2 l_{\max }\right] \times 32 N$.)

It is important that the density of the points is sufficiently high so that they can capture the highest-frequency components of the spherical harmonics ( to visualize the induced electric field (or the current density), for example if the points are chosen uniformly distributed on the sphere.

Choosing the cortex radius to be $r_{\text {target }}=70 \mathrm{~mm}$, the coil radius to be $R_{\text {current }}=90 \mathrm{~mm}$, and the cut-off point for the spherical harmonic series to be $l_{\max }=30$; we get a convex cost function with $\left(l_{\max }\right)^{2}+2 l_{\max }=960$ input variables. With this target radius and surface current radius, the radius for head can be, for example, $85 \mathrm{~mm} .{ }^{4}$ These radii are the same that were used by Deng et al. in their study of 50 different TMS-coil designs [2]. Thus, this choice allows a direct comparison between the results.

\footnotetext{
${ }^{3} \mathrm{~A}$ feasible point is a point that fulfills all the constraints.

${ }^{4}$ Like in the method by Eaton, [8], there is no direct dependence on the radius of head in this model.
} 


\subsection{Discretization of the surface current density}

Surface current densities like those described in the previous chapter can not be realized in practice. However, they can be approximated with a coil consisting of several winding loops, and this coil can be used as the TMS coil. A surface current density can be discretized using stream functions [19], $\psi$, for which

$$
\boldsymbol{K}=\nabla \psi \times \boldsymbol{n},
$$

where $\boldsymbol{K}$ is the surface current density and $\boldsymbol{n}$ is the normal vector of the surface. The stream functions for real vector spherical harmonics can be seen directly from Eq. (A.10):

$$
\boldsymbol{Y}_{l, l}^{m}(\theta, \phi)=\frac{1}{\sqrt{l(l+1)}} \boldsymbol{r} \times \nabla Y_{l}^{m}(\theta, \phi)=\nabla\left[-\frac{r}{\sqrt{l(l+1)}} Y_{l}^{m}(\theta, \phi)\right] \times \boldsymbol{e}_{r} .
$$

Thus, the stream function for the surface current of the form

$$
\boldsymbol{K}(\theta, \phi)=\sum_{l, m} i_{l m} \boldsymbol{Y}_{l, l}^{m}(\theta, \phi) / R
$$

is

$$
\psi(\theta, \phi)=-\sum_{l, m} \frac{i_{l m}}{\sqrt{l(l+1)}} Y_{l}^{m}(\theta, \phi) .
$$

Next, we select the number of steps used to approximate the stream function, $N_{\text {steps. }}$. For simple coil designs, such as circular or figure-of-eight coil, this is also the number of turns in the coil. However, for more complicated designs, the numbers of turns in the coil may be higher. Now, we can define the coil current $^{5}$

$$
I=\frac{\max \psi-\min \psi}{N_{\text {steps }}} .
$$

The coil loops are on the isolines of $\psi$ such that

$$
\psi(\theta, \phi)=\min \psi+\left(n-\frac{1}{2}\right) I, n \in\left\{1, \ldots, N_{\text {steps }}\right\} .
$$

The loops can be further discretized into polygons, for which the inductances can be calculated using formulas from Ref. [20].

The induced electric field inside the head can be calculated using the triangle construction described earlier in Section 2.3 (i.e., by calculating the mutual inductance of the coil and a triangle). However, now the triangle width has to be finite (not infinitesimal). We used a width of $0.02 \mathrm{rad} \cong 1.4 \mathrm{~mm}$ for these triangles.

\subsection{Measurement set-up}

In order to validate the computational methods, we manufactured one optimized coil design. The coil was wound with 1-mm-thick continuous copper wire

\footnotetext{
${ }^{5}$ This is not exactly the same as the current required for the stimulus because the discretized surface current density causes a different stimulation pattern in the cortex than the continuous surface current density. This is apparent when $N_{\text {steps }}$ is small.
} 
by connecting the separate loops into each other in a spiral-like fashion. To achieve sufficient winding precision, we fabricated a coil former using additive manufacturing. The groove for the wire was made snug, which allowed fast glue-free assembly. The reader should be aware that this prototype is not a practical TMS coil as it was only intended for validating the computational methods. For an actual TMS coil, one should, for example, glue the wire to the coil former because of the Lorenz forces that are induced by the TMS pulse (although these force are smaller in the optimized coil design than in standard figure-of-eight coils). The coil was connected in series with a signal generator for the measurements.

In order to measure the induced electric field, a computer-controlled measurement rig was built [21]. The rig consists of two orthogonal triangular loops for the triangle construction, and two servo motors to control the orientation of the loops. The triangle loops are wound with a 0.15 -mm-diameter copper wire on a 3D-printed coil former; they have 70-mm-long radial edges and a 5-mm-long tangential edge. This non-differential tangential edge means that the construction can measure the higher-frequency components of the spherical harmonics (sinusoidal waves with angular frequency $\pm l_{\max }$ ) components with up to

$$
l_{\max } \approx \frac{2 \pi \cdot 70 \mathrm{~mm}}{2 \cdot 5 \mathrm{~mm}} \approx 40 .
$$

This is enough to capture all details of the induced electric field at this depth, as higher than this spatial-frequency components will have attenuated by five orders of magnitude more than the $(l=1)$ components. The coil and the measurement rig are shown in Fig. 3.

The measurement points are chosen pseudo-uniformly from the upper hemisphere. With 1000 points, the mean distance to the nearest neighbor is $5.3 \mathrm{~mm}$ (and the largest single distance $5.5 \mathrm{~mm}$ ). Thus, all the spatial-frequency components that can reach the target depth are easily recorded.

The spatial accuracy of the servos used in the rig is approximately $2^{\circ} \cong$ $2.5 \mathrm{~mm}$ and the relative standard error between the two channels in the induced electric field measurement is approximately $2 \%$ after a calibration with a circular current loop. 


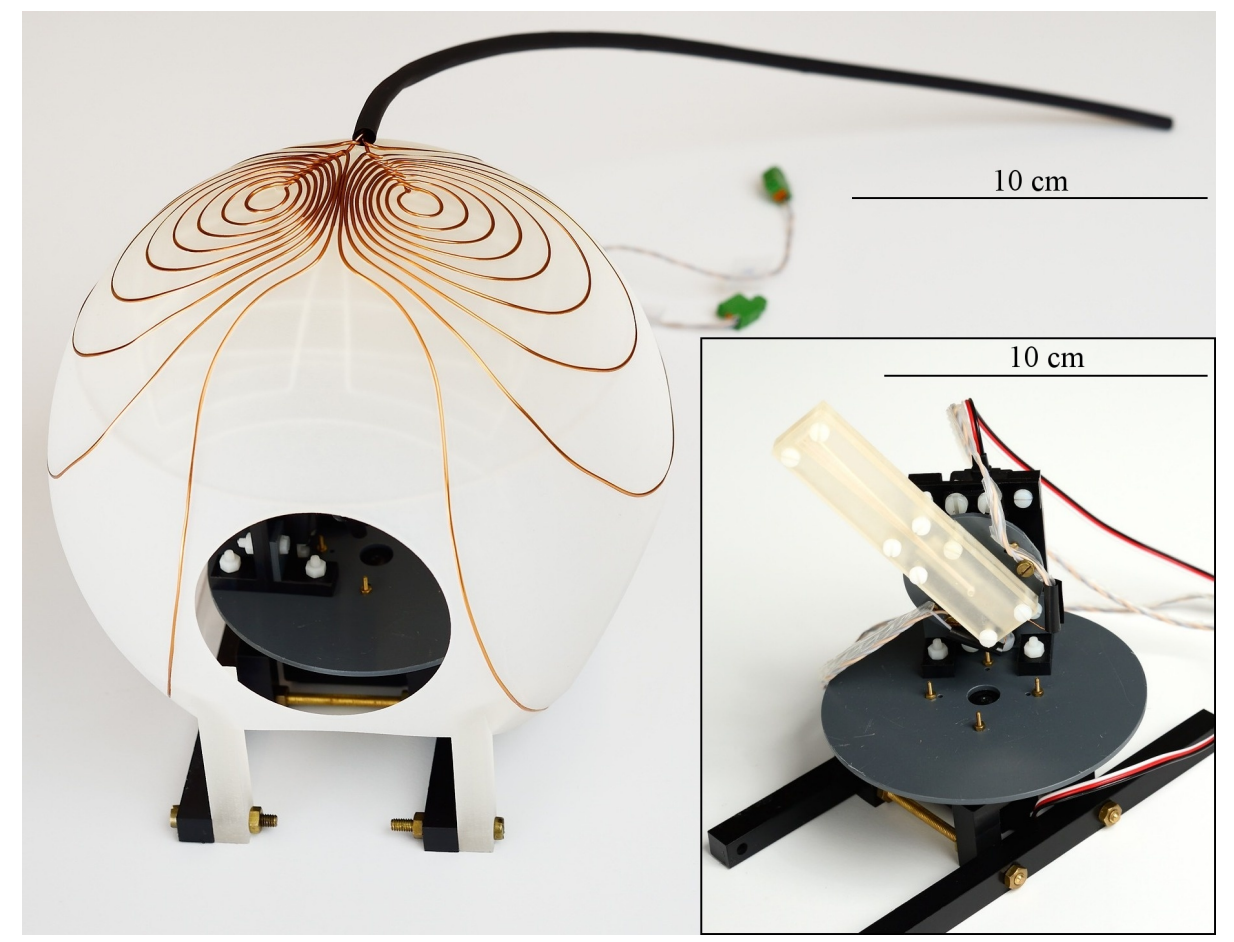

Figure 3: The minimum-energy TMS coil used to validate the computational methods. The coil is wound on a plastic spherical coil former, which was 3Dprinted. The coil was only used to validate the methods and it is not a practical TMS coil. The measurement rig (inset) fits inside the hollow coil former. 


\section{Results}

Using the constraints from Section 3.2 and the parameters from Section 3.3, we could solve for the global optimum solution with different focalities. The dependency between the minimum energy and the parallel and perpendicular focalities is shown in Fig. 4. In this plot, we show the smallest peak magneticfield energy for a $100-\mu$ s $100-\mathrm{V} / \mathrm{m}$ stimulus.

We chose Magstim $70 \mathrm{~mm}$ Double Coil as a baseline for the comparison. The reason for this is that the structure of the Magstim coil is depicted in Ref. [22]. Approximating the coil with 1-mm-thick wiring at a plane $90 \mathrm{~mm}$ from the origin, we obtain for it an inductance of $15.90 \mu \mathrm{H}$, which is close to specifications $(16.35 \mu \mathrm{H})$. We refer to this coil as a standard figure-of-eight coil, or the standard coil.

The FWHM for the standard figure-of-eight coil was $32 \mathrm{~mm}$ in the direction perpendicular to the electric field and $50 \mathrm{~mm}$ in the direction parallel with it. A $100-\mu \mathrm{s} 100-\mathrm{V} / \mathrm{m}$ pulse with the standard coil required maximum current of $6400 \mathrm{~A}$ and thus coil energy of $330 \mathrm{~J}$. The coil and the resulting E-field are visualized in Fig. 5a. The optimal solution for this kind of stimulus required just $77 \mathrm{~J}$ of stored energy, thus we can reduce the energy requirement significantly or alternatively make the stimulus more focal with the original energy requirement.

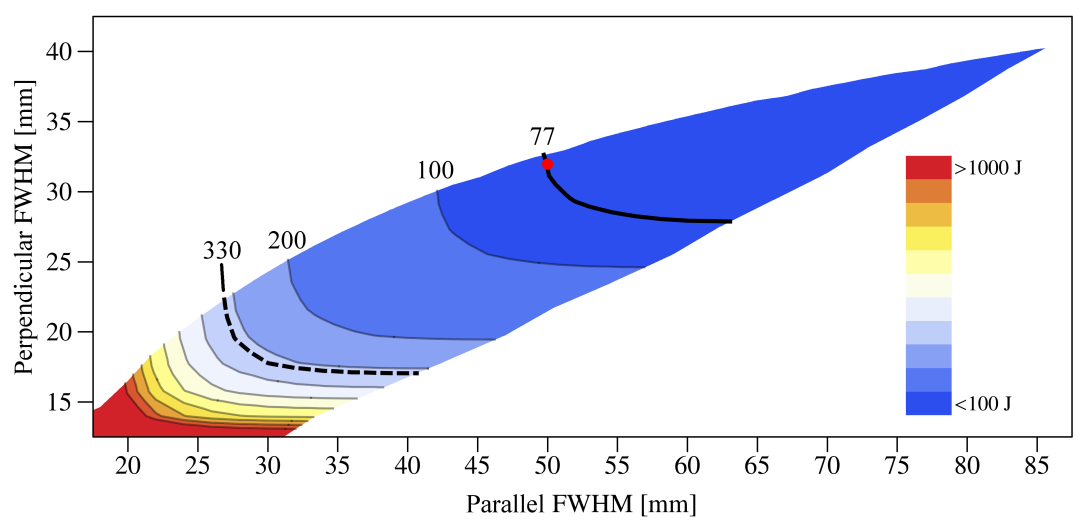

Figure 4: Minimum energy for given FWHM. Trying to deviate the ratio of parallel and perpendicular FWHM far from optimal ratio (of approximately $2: 1$ ) will require more energy, and thus in the region painted white, it is beneficial to reduce the FWHM instead of increasing it. (Also, when trying to produce a stimulus with broader focality than $85 \mathrm{~mm}$ parallel FWHM and $40 \mathrm{~mm}$ perpendicular FWHM, broadening the focality will require more energy instead of less energy.) The solid black line is the 77-J contour line and the dashed black line the 330-J contour line. The other contour lines are drawn at multiples of $100 \mathrm{~J}$, and the red point represents the FWHM of the standard coil.

\subsection{Discretization of the surface current density}

Discretization of the surface current densities allows using other field-computation methods to verify the obtained results and to compare them to values from existing TMS coils. 
Solving the optimization problem from Section 3.2 with the parameters from Section 3.3 with the focality of the standard coil yielded a butterfly-like continuous surface current distribution. Using this, the $100-\mu \mathrm{s} 100-\mathrm{V} / \mathrm{m}$ pulse had a maximum energy of (only) $77 \mathrm{~J}$. Then, this current density was discretized into 18 loops (the same number as in the standard coil). The resulting coil had an inductance of $7.8 \mu \mathrm{H}$. For a $100-\mu \mathrm{s} 100-\mathrm{V} / \mathrm{m}$ pulse this coil required maximum current of $4800 \mathrm{~A}$ and thus maximum coil energy of $89 \mathrm{~J}$. The coil and the resulting E-field are visualized in Fig. 5b.

We can see from Fig. 5 that the induced electric fields for the standard and the optimized coil are similar near the focal point. However, the optimized design has a higher E-field further away from the focal point. This phenomenon is visualized in Fig. 6.

If we calculate the volume of the area inside the brain, where $|\boldsymbol{E}| \geq\left|\boldsymbol{E}_{0}\right| / 2$, we obtain $V_{1 / 2}$, standard $=23.9 \mathrm{~cm}^{3}$ and $V_{1 / 2 \text {, optimized }}=37.1 \mathrm{~cm}^{3}$. The corresponding maximum depths, where this condition is met are $d_{1 / 2}$ standard $=$ $1.35 \mathrm{~cm}$ and $d_{1 / 2}$, optimized $=1.72 \mathrm{~cm}$. Using Eq. (15) results in $S_{1 / 2}$, standard $=$ $17.6 \mathrm{~cm}^{2}$ and $S_{1 / 2}$, optimized $=21.6 \mathrm{~cm}^{2}$. Thus, although the coils are equally focal using our proposed focality metric, the optimized coil design is significantly less focal than the standard coil using the focality metric by Deng et al. This difference here is mostly because we defined the focality as $1 / \sqrt{2}$-maximum instead of $1 / 2$-maximum. If we replace the boundary conditions in the optimization problem with 50\%-boundary conditions obtained from the standard-coil, we will obtain a 83-J continuous surface current density. This can be discretized into a 95-J coil, for which $d_{1 / 2}=1.40 \mathrm{~cm}$ and $S_{1 / 2}=15.8 \mathrm{~cm}^{2}$ (Fig. 5c).

In addition to these coils, we also created a very focal 690-J continuous current density (with parallel FWHM of $22 \mathrm{~mm}$ and perpendicular FWHM of $18 \mathrm{~mm}$ ). From this, we obtain a coil with an inductance of $5.1 \mu \mathrm{H}$, maximum current of $16000 \mathrm{~A}$, and maximum coil energy of 690 J. For this coil, the parallel FWHM is $24 \mathrm{~mm}$ and perpendicular FWHM is $18 \mathrm{~mm}$ (Fig. 5d).

\subsection{Comparing the calculated and measured values}

An optimized current distribution with perpendicular FWHM of $31 \mathrm{~mm}$ and parallel FWHM of $51 \mathrm{~mm}$ was computed. This was then discretized into 18 current loops, which were modeled as 1-mm-thick wires. The inductance of this coil was computed to be $8.07 \mu \mathrm{H}$. Let us define gain as the ratio between maximum induced electric field and the time-derivative of current,

$$
\text { gain }=\frac{E_{0}}{d I / d t} .
$$

The computed gain for the coil is $2.13(\mu \mathrm{V} / \mathrm{m}) /(\mathrm{A} / \mathrm{s})$, which gives for $100-\mu \mathrm{s}$ pulse $U_{\max }=\frac{1}{2} L I^{2}=89 \mathrm{~J}$.

The individual current loops were connected into a single coil, and an actual coil was wound from 1-mm copper wire. This coil is shown in Fig. 3. Measuring the E-field using the measurement rig from Section 3.5, we obtain perpendicular FWHM of $33 \mathrm{~mm}$ and the parallel FWHM of $51 \mathrm{~mm}$. The resistance of the coil was $87 \mathrm{~m} \Omega$, the inductance at $10 \mathrm{kHz}$ was $8.14 \mu \mathrm{H}$, and the maximum gain was $2.01(\mu \mathrm{V} / \mathrm{m}) /(\mathrm{A} / \mathrm{s})$, which give $U_{\max }=100 \mathrm{~J}$. In Fig. 7 , we have plotted the measured gain versus the calculated gain at 1000 points inside the head. The 

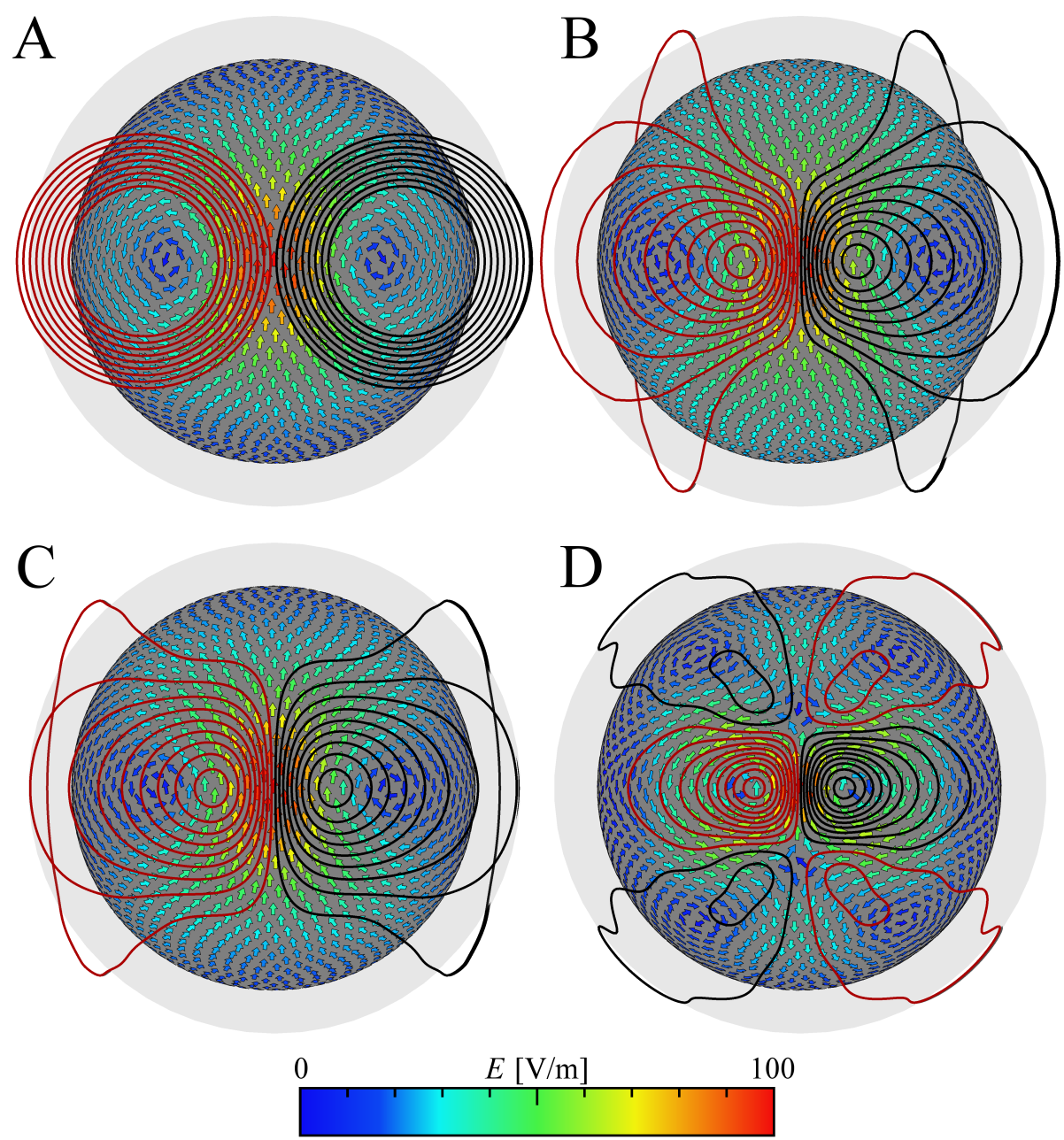

Figure 5: The coil geometry and the induced electric field for (A) the standard figure-of-eight coil, (B) the optimized coil, (C) the 50\%-boundary-conditionoptimized coil, and (D) a very focal coil. (All numbers calculated assuming a 100- $\mathrm{ss}$ TMS pulse with $100-\mathrm{V} / \mathrm{m}$ maximum induced electric field. The current direction is clockwise in the red wires and counterclockwise in the black wires.)

least-squares fit between these two indicate that the measured gain was $6 \%$ smaller than the computed one. 

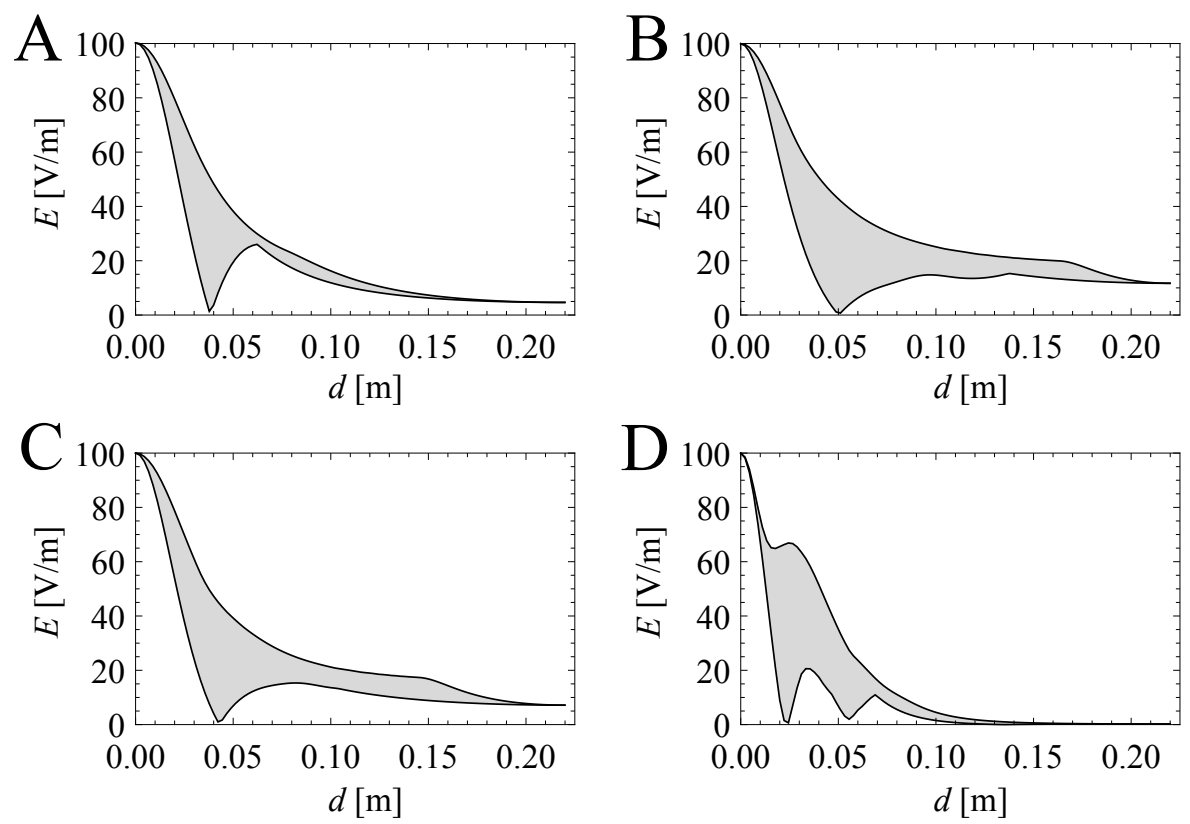

Figure 6: Envelopes of the electric field strength as a function of distance $d$ from the target point. The upper black line represents the maximum E-field in any direction and the lower black line represents the minimum E-field in any direction. The standard coil and the two optimized coils have very similar E-fields near the target. (A) standard coil; (B) optimized design; (C) 50\%boundary-condition-optimized design; (D) a very focal coil (coils A-D, Fig. 5). 


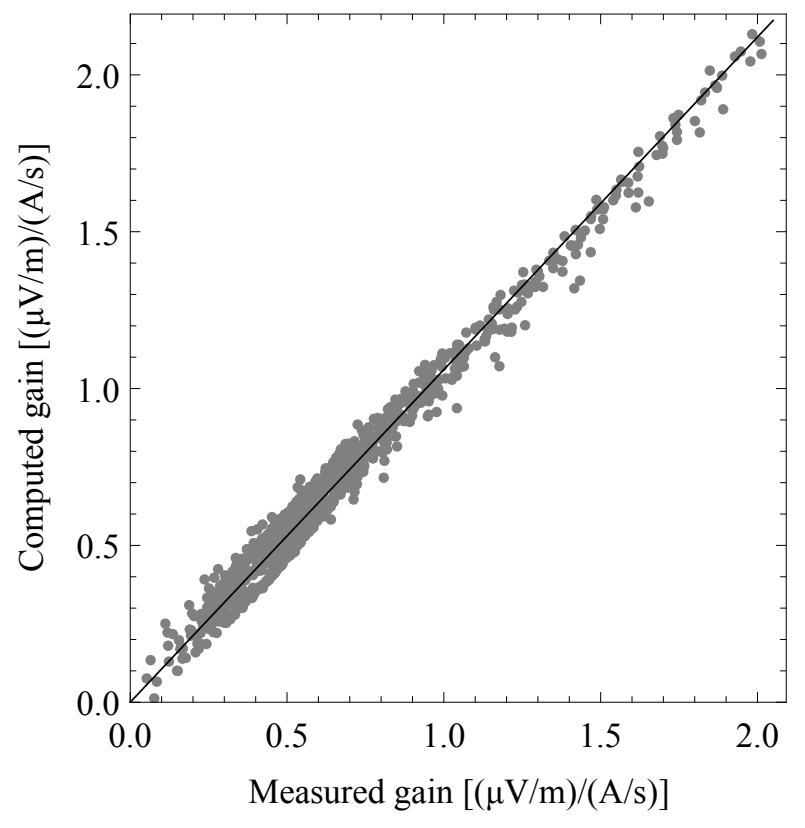

Figure 7: Comparizon between the measured gain and the calculated gain for the coil in Fig. 3. The line has a slope of 1.060. The Pearson correlation coefficient between the two data sets is 0.991 . The spread in the data is mostly due to small systematic positional errors of the measurement probe. 


\section{Discussion}

The optimal coils in Fig. 5 resemble the optimized muscle stimulation coil from Ref. [23]. In both cases, the winding density has been concentrated over the stimulation point, and the coil has been curved to follow the surface of the tissue. As they optimized their coils in a cylinderical geometry, it seems that general features of the minimum-energy coils are robust to the tissue geometry. A somewhat similar coil design was also evaluated in Ref. [2] (coil \#38), where its E-field was found to resemble that of a figure-of-eight coil.

The induced electric field patterns of the optimized coil geometries (Figs. 5b and 5c) are similar to that of the standard figure-of-eight coil near the focal region. That is, these three coils will produce almost identical brain stimulation near the target. However, the optimized coils cause a much larger induced electric field in the region opposite to the target. For the first geometry, the induced electric field near the opposite point of the head is on average $20 \%$ of the maximum induced electric field; twice that of the standard coil. Still, this should not significantly affect the outcome of a stimulus as an induced electric field with $20 \%$ of the maximum strength will cause little activation of the cortex. Thus, the optimized coils will stimulate the brain in a very similar manner to the standard coil. Because different figure-of-eight coils seem to have similar field characteristics $[2,15]$, we can say that the optimized designs behave like any 70 -mm figure-of-eight coil.

However, there are some practical considerations with the optimized design shown in Fig. 5b. First, the farthest-away wires extend almost to the opposite side of the head. This limits the possible regions of head that can be reached, as these wires would have to go below the chin. The problem can be addressed in two ways. The naive approach is to deform the wires away from the spherical shape; this will increase the inductance. The other solution is to add constraints also for the current distribution; we can for example demand that the surface current density is small at the region opposite to the target. This will increase the proportion of the higher order terms, and thus increase the needed current.

As the radial coil current elements do not contribute to the stimulation, the induced E-field of, for example, the slinky coil [5] can also be realized with a more efficient flat coil. Because a circular coil held perpendicular to the head surface produces an induced E-field similar to that of a figure-of-eight coil, the flat version of the slinky coil will resemble a figure-of-eight coil with the windings concentrated over the target point (i.e., similar to the optimized coil).

Also, for example, the very focal coil in Fig. 5d is topologically equivalent to the 3D differential coil proposed by Hsu and Durand in Ref. [24]. That is, in both coils there are two additional wings with opposite current direction to the main part of the coil reducing the region of high E-field in the perpendicular direction. The major difference between these coil designs is that in their coil design the wings were implemented in an inefficient manner with perpendicularto-head circular coils.

In a recent paper, different designs for deep TMS (dTMS) were evaluated [25]. The coil focality was shown to deteriorate with stimulus depth, and closeto-head coils were found more efficient than 3D coil designs such as the H1 Hesed coil. These findings are in agreement with our findings: the focality is reduced because the higher spatial frequencies decay faster than lower spatial frequencies when the stimulus depth is increased, and because having radial 
current elements in the coil is inefficient as there exists a more efficient, closeto-head, coil producing the same induced E-field as that of any 3D coil design. The methods from our paper can also be applied for optimizing dTMS coils.

In this paper, we have only considered an air coil, i.e., a coil without an iron core. In Ref. [26], Epstein and Davey showed how an iron-core coil can be significantly more efficient than an air coil. By suppressing the external magnetic fields, an iron core can further reduce the energy of the minimumenergy solution with given focality constraints.

\subsection{Definition of the focality}

The definition of the focality is ambiguous. In the literature, the focality of a stimulus is often determined based on the surface area or the volume where the stimulus intensity is greater than $50 \%$ of the maximum. The boundary of this region is usually relatively far from the target point. Thus, this does not necessarily work well for estimating the focality of a relatively focal stimulus.

On the other hand, a design that is equally focal according to the 50\%boundary metric is not necessarily equally focal according to the $70 \%$-boundary metric and the other way round. This can be seen from the coil designs of Fig. 5 . Although the two coils (Figs. 5a and 5b) are, by design, similar according to our focality metric, they differ significantly in the focality metric by Deng et al., Ref. [2]. Because of this, one should select the focality metric based on the application of the stimulus.

For example, if one is to design a coil for mapping the motor cortex with pulses just above the motor threshold, one should use a metric where the focality is defined with a high threshold boundary. The extreme case of this would be using the spatial curvature of the electric field strength, that is, the second spatial derivatives of the pulse strength at the target. 


\section{Conclusion}

We have formulated a model for computing the induced electric field in the spherically symmetric head model for an arbitrary spherical surface current density. Using this model, the global optimum solution for low-depth TMS coil is an as-close-to-head-as-possible double-coned coil with butterfly-wing shaped windings (Fig. 5b). This design is significantly better than the standard figureof-eight solution.

The focality of a TMS coil can be increased quite significantly from the current designs. This can be achieved although reducing perpendicular FWHM from $30 \mathrm{~mm}$ to $20 \mathrm{~mm}$ increases the required energy threefold. ${ }^{6}$ This is because the present coil designs seem to be far from optimal, the optimized coil from Fig. $5 \mathrm{~b}$ requires $73 \%$ less energy for producing similar stimulation as the standard coil (Fig. 5a). This is due to the fact that in-plane figure-of-eight design has both greater inductance and worse connection to the cortex than the closeto-head-everywhere design.

We were able to verify the results experimentally. The calculation and the measurement gave, within measurement accuracy, the same FWHMs. Though the measured gain differs from the calculated gain by $6 \%$, which is much more than the measurement accuracy of $2 \%$ between the two channels, the shape of the induced electric field is similar for both calculation and the measurement. Thus, this difference may be partially due to uncertainty in the calibration process. However, the most likely explanation for this error is inaccuracy in the coil position and the coil windings with respect to the measurement probe. Namely, our simulations reveal that an error of $1 \mathrm{~mm}$ in the coil position radial to the focal point changes the induced E-field magnitude by $4 \%$.

In summary, there is room for improvements from the present coil designs. The simple figure-of-eight design needs approximately four times more energy than the theoretical limit for producing a similar induced electric field. With our methods, we could design and produce an optimized coil that is less than one third more expensive than the obtained theoretical limit.

\section{Acknowledgement}

This research received funding from the Academy of Finland. The authors thank Mika Salmi for helping with the fabrication of the 3D-printed parts and Ilkka Nissilä for the photographs.

Conflict of interest statement. Risto Ilmoniemi is an advisor and a minority shareholder of Nexstim Ltd. The other authors declare no conflict of interest.

\footnotetext{
${ }^{6}$ The exact number depends on the target depth-here it is $20 \mathrm{~mm}$ as the coils are placed at the height of $90 \mathrm{~mm}$ and the target radius is $70 \mathrm{~mm}$. For slightly farther targets at $r_{\text {target }}=$ $60 \mathrm{~mm}$, a similar increase in focality requires more than fivefold more energy.
} 


\section{A Mathematical tools}

\section{A.1 Basic electromagnetism}

The fourth Maxwell equation-Ampère's circuit law with Maxwell's correctionstates that

$$
\nabla \times \boldsymbol{B}=\mu_{0} \boldsymbol{J}+\mu_{0} \varepsilon_{0} \frac{\partial \boldsymbol{E}}{\partial t},
$$

where $\boldsymbol{B}$ is the magnetic field, $\mu_{0}$ is the vacuum permeability, $\boldsymbol{J}$ is the current density, $\varepsilon_{0}$ is the vacuum permittivity, and $\boldsymbol{E}$ is the electric field. In the quasistatic case in a region where there is no source current density [27], ${ }^{7}$ Eq. (A.1) can be written as

$$
\nabla \times \boldsymbol{B}=0
$$

Thus, the magnetic field may be represented with the magnetic scalar potential $V$ :

$$
\boldsymbol{B}=-\mu_{0} \nabla V \text {. }
$$

The second Maxwell equation-Gauss's law for magnetism - states that

$$
\nabla \cdot \boldsymbol{B}=0
$$

Combining Eqs. (A.3) and (A.4) we get Laplace's equation:

$$
\nabla^{2} V=0
$$

\section{A.2 Real spherical harmonics}

The general solution of Eq. (A.5) in spherical coordinates $(r, \theta, \phi)$ is

$$
V(r, \theta, \phi)=\sum_{l=0}^{\infty} \sum_{m=-l}^{l}\left[a_{l m} r^{l}+b_{l m} r^{-(l+1)}\right] Y_{l}^{m}(\theta, \phi)
$$

where $Y_{l}^{m}(\theta, \phi)$ are real spherical harmonics and $a_{l m}$ and $b_{l m}$ are constants. The real spherical harmonics are defined as

$$
Y_{l}^{m}(\theta, \phi)= \begin{cases}\sqrt{\frac{(2 l+1)(l-m) !}{2 \pi(l+m) !}} P_{l}^{m}(\cos \theta) \cos m \phi, & m>0 \\ \sqrt{\frac{2 l+1}{4 \pi}} P_{l}^{0}(\cos \theta) & m=0 \\ -\sqrt{\frac{(2 l+1)(l+m) !}{2 \pi(l-m) !}} P_{l}^{-m}(\cos \theta) \sin m \phi, & m<0\end{cases}
$$

where $P_{l}^{m}(x)$ are associated Legendre polynomials of degree $l$ and order $m$. Just like the complex spherical harmonics, the real spherical harmonics are orthonormal over the sphere:

$$
\int_{4 \pi} Y_{l}^{m} Y_{L}^{M} d \Omega=\delta_{l L} \delta_{m M}
$$

\footnotetext{
${ }^{7}$ Inside the head, there are obviously currents, but these are much weaker than the coil currents. Thus, for our purposes, it suffices to calculate the magnetic field due to the TMS coil.
} 


\section{A.3 Real vector spherical harmonics}

Because we are interested in the magnetic field, it is useful to define vectorvalued spherical harmonics. These can be defined as [28]

$$
\begin{aligned}
\boldsymbol{Y}_{l, l+1}^{m}(\theta, \phi) & =\frac{1}{\sqrt{(l+1)(2 l+1)}} r^{l+2} \nabla\left[\frac{1}{r^{l+1}} Y_{l}^{m}(\theta, \phi)\right], & & l \geq 0, \\
\boldsymbol{Y}_{l, l}^{m}(\theta, \phi) & =\frac{1}{\sqrt{l(l+1)}} \boldsymbol{r} \times \nabla Y_{l}^{m}(\theta, \phi), & & l \geq 1, \\
\boldsymbol{Y}_{l, l-1}^{m}(\theta, \phi) & =\frac{1}{\sqrt{l(2 l+1)}} \frac{1}{r^{l-1}} \nabla\left[r^{l} Y_{l}^{m}(\theta, \phi)\right], & & l \geq 1 .
\end{aligned}
$$

The notation allows a direct comparison between the complex vector spherical harmonics introduced in Refs. [29, 30]. These vector-valued functions are orthonormal over the sphere:

$$
\int_{4 \pi} \boldsymbol{Y}_{l, l+n}^{m} \cdot \boldsymbol{Y}_{L, L+N}^{M} d \Omega=\delta_{l L} \delta_{m M} \delta_{n N} ; n, N \in\{-1,0,1\} .
$$

Furthermore, the real vector spherical harmonics form a complete set and can present any vector function on the surface of a sphere. The following properties of the vector spherical harmonics are used in Appendix B:

$$
\nabla \times\left(f \boldsymbol{Y}_{l, l}^{m}\right)=\sqrt{\frac{l}{2 l+1}}\left(\frac{l}{r} f-\frac{\partial f}{\partial r}\right) \boldsymbol{Y}_{l, l+1}^{m}-\sqrt{\frac{l+1}{2 l+1}}\left(\frac{l+1}{r} f+\frac{\partial f}{\partial r}\right) \boldsymbol{Y}_{l, l-1}^{m}
$$

and

$$
\nabla \times\left(\nabla \times f \boldsymbol{Y}_{l, l}^{m}\right)=\left(\frac{l(l+1)}{r^{2}}-\frac{2}{r} \frac{\partial}{\partial r}-\frac{\partial^{2}}{\partial r^{2}}\right) f \boldsymbol{Y}_{l, l}^{m},
$$

where $f(r)$ is a scalar function. Many other identities for the real vector spherical harmonics can also be obtained by adapting those given in Ref. [30].

\section{A.4 Divergence-free basis functions}

Following the derivation in Ref. [31], it is possible show that an arbitrary divergence-free real-valued vector function in a volume can be presented as a linear combination of the basis functions

$$
\boldsymbol{u}_{l m}(r, \theta, \phi)=f_{l m}^{-}(r) \boldsymbol{Y}_{l, l}^{m}(\theta, \phi)+\nabla \times\left(f_{l m}^{+}(r) \boldsymbol{Y}_{l, l}^{m}(\theta, \phi)\right),
$$

where $f_{l m}^{-}$and $f_{l m}^{+}$are functions defining the radial dependency. $f_{l m}^{-}$and $f_{l m}^{+}$can also be presented using a complete set of elementary scalar functions satisfying the boundary conditions. Note that, on a spherical shell, we can form a complete set of basis functions for divergence-free vector fields by the real vector spherical harmonics of Eq. (A.10). 


\section{B Proof of the optimality of a single-layer cur- rent density}

In this Appendix, we provide a proof that a single current-carrying layer placed as close to the spherically symmetric target volume ("head") as possible is the optimal solution in the sense that it minimizes the inductive energy.

First, let us consider current density that has no radial components. We will show that it is always beneficial to place all such tangential currents as close to the head as possible.

Let us consider current density at two spherical shells with radii $R_{1}$ and $R_{2}>R_{1}$. We can compute the energy stored in the magnetic field caused by these two shells using the Eqs. (1), (7), and (8). This energy can be divided into two components, the sum of the energies of the two shells alone (Eq. (9)) and a cross-term from their interaction with each other:

$$
U=\left(U_{1}+U_{2}\right)+\tilde{U} .
$$

From the orthonormality of the real vector spherical harmonics (Eq. (A.12)), we see that the surface integral over the cross-term energy density is zero if $l_{1} \neq l_{2}, m_{1} \neq m_{2}$, or $R_{1}<r<R_{2}$. Thus, the energy stored in the cross terms of the magnetic field of the two spherical shells is

$$
\tilde{U}=\mu_{0} \sum_{l, m} i_{1, l m} i_{2, l m}\left[\int_{0}^{R_{1}} \frac{l+1}{2 l+1} \frac{r^{2 l-2}}{R_{1}^{l} R_{2}^{l}} r^{2} d r+\int_{R_{2}}^{\infty} \frac{l}{2 l+1} \frac{R_{1}^{l+1} R_{2}^{l+1}}{r^{2 l+4}} r^{2} d r\right],
$$

where the constants $i_{1, l m}$ define the current distribution in the inner shell and $i_{2, l m}$ in the outer shell. Defining $R_{2}:=\alpha R_{1}=\alpha R, \alpha \geq 1$, we obtain

$$
\begin{aligned}
\tilde{U} & =\mu_{0} \sum_{l m} i_{1, l m} i_{2, l m}\left[\int_{0}^{R} \frac{l+1}{2 l+1} r^{2 l} R^{-2 l} \alpha^{-l} d r+\int_{\alpha R}^{\infty} \frac{l}{2 l+1} r^{-2 l-2} R^{2 l+2} \alpha^{l+1} d r\right] \\
& =\mu_{0} \sum_{l m} i_{1, l m} i_{2, l m}\left[\frac{l+1}{2 l+1} \frac{1}{2 l+1} R \alpha^{-l}+\frac{l}{2 l+1} \frac{1}{2 l+1} R \alpha^{-l}\right] \\
& =\sum_{l m} \frac{\mu_{0} R}{(2 l+1) \alpha^{l}} i_{1, l m} i_{2, l m} .
\end{aligned}
$$

From Eq. (13), we obtain the induced electric field. For two shells, we have

$$
\boldsymbol{E}(\theta, \phi)=-\mu_{0} \sum_{l m} \frac{\partial f(t)}{\partial t} \frac{1}{2 l+1} \boldsymbol{Y}_{l, l}^{m}(\theta, \phi)\left[\left(\frac{r}{R}\right)^{l} i_{1, l m}+\left(\frac{r}{\alpha R}\right)^{l} i_{2, l m}\right]
$$

and

$$
U=\frac{1}{2} \sum_{l m} \frac{\mu_{0} R}{2 l+1}\left[i_{1, l m}^{2}+2 \frac{i_{1, l m} i_{2, l m}}{\alpha^{l}}+\alpha i_{2, l m}^{2}\right] .
$$

For given $\boldsymbol{E}$, these equations are equivalent to

$$
\begin{aligned}
& \text { constant } \equiv E_{l m}^{\prime}=i_{1, l m}+\alpha^{-l} i_{2, l m}, \\
& U_{l m}^{\prime}\left(i_{1, l m}, i_{2, l m}\right)=i_{1, l m}^{2}+2 \frac{i_{1, l m} i_{2, l m}}{\alpha^{l}}+\alpha i_{2, l m}^{2},
\end{aligned}
$$


and

$$
U^{\prime}=\sum_{l, m} U_{l m}^{\prime}
$$

Here, we want to minimize $U^{\prime}$, which is achieved by minimizing the Lagrangian $\Lambda_{l m}$ :

$$
\Lambda_{l m}\left(i_{1, l m}, i_{2, l m}, \lambda\right)=U_{l m}^{\prime}\left(i_{1, l m}, i_{2, l m}\right)+\lambda_{l m}\left(i_{1, l m}+\alpha^{-l} i_{2, l m}-E_{l m}^{\prime}\right),
$$

where $\lambda_{l m}$ are the Lagrange multipliers.

$$
\nabla \Lambda_{l m}\left(i_{1, l m}, i_{2, l m}, \lambda_{l m}\right)=0 \Leftrightarrow\left\{\begin{array}{l}
2 i_{1, l m}+2 \alpha^{-l} i_{2, l m}+\lambda_{l m}=0 \\
2 \alpha^{-l} i_{1, l m}+2 \alpha i_{2, l m}+\lambda_{l m} \alpha^{-l}=0 \\
i_{1, l m}+\alpha^{-l} i_{2, l m}-E_{l m}^{\prime}=0
\end{array} .\right.
$$

The solution to this is $i_{1, l m}=E_{l m}^{\prime}, i_{2, l m}=0$, and $\lambda_{l m}=-2 E_{l m}^{\prime}$. This is equivalent with the situation that all the current density moved to the inner shell.

Although we only examined a two-shell system, the result holds for an arbitrary number of shells. This is because it is beneficial to move the currents one by one to the innermost shell using the following procedure:

1. Take the two innermost shells.

2. Combine the second with the first. This reduces the connection of that shell with the remaining shells (because the coupling (Eq. (B.5)) between the remaining shells is reduced) and enhances the coupling with the brain.

3. Repeat the process until you have only one shell left.

The continuum of radial shells can be thought of as a limiting procedure.

Next, we will examine the effect of radial current densities on the energy efficiency of the stimulation. Given the basis functions of Eq. (A.15), let us consider divergence-free volume current density of the form

$$
\boldsymbol{J}_{l m}(r, \theta, \phi)=\left\{\begin{array}{ll}
0, & r<R \\
\nabla \times\left(j_{l m}(r) \boldsymbol{Y}_{l, l}^{m}(\theta, \phi)\right), & r \geq R
\end{array},\right.
$$

where $j_{l m}(r)$ is a function defining the radial dependency of the current density. At the boundary, the continuity of the current requires $j_{l m}(R)=0$. We can solve the corresponding magnetic field $\boldsymbol{B}_{l m}$ by noting that $\nabla \cdot \boldsymbol{B}_{l m}=0$ and $\nabla \times \boldsymbol{B}_{l m}=\mu_{0} \boldsymbol{J}_{l m}$. Thus, because $\boldsymbol{B}_{l m}$ is also divergence-free, we can express it using the basis functions of Eq. (A.15):

$$
\boldsymbol{B}_{l m}(r, \theta, \phi)=\left\{\begin{array}{ll}
a_{l m, \text { int }}(r) \boldsymbol{Y}_{l, l}^{m}(\theta, \phi)+\nabla \times\left(b_{l m, \text { int }}(r) \boldsymbol{Y}_{l, l}^{m}(\theta, \phi)\right), & r<R \\
a_{l m, \text { ext }}(r) \boldsymbol{Y}_{l, l}^{m}(\theta, \phi)+\nabla \times\left(b_{l m, \text { ext }}(r) \boldsymbol{Y}_{l, l}^{m}(\theta, \phi)\right), & r \geq R
\end{array},\right.
$$

where $a_{l m, \text { int }}, a_{l m, \text { ext }}, b_{l m, \text { int }}$, and $b_{l m, \text { ext }}$ define how the magnetic field behaves as a function of $r$. Because of Eqs. (A.12), (A.13), and (A.14), the magnetic field should have the same $(l, m)$ indices as the current density. Requiring that $\nabla \times \boldsymbol{B}_{l m}=\mu_{0} \boldsymbol{J}_{l m}$, evaluating the curls by using Eqs. (A.13) and (A.14), and 
requiring that the multipliers of the vector spherical harmonics in the resulting equations are zero, we obtain the following set of differential equations:

$$
\begin{aligned}
\frac{l}{r}\left(\mu_{0} j_{l m}(r)-a_{l m, \text { ext }}(r)\right)+\frac{\partial}{\partial r}\left(a_{l m, \text { ext }}(r)-\mu_{0} j_{l m}(r)\right) & =0 \\
\frac{l+1}{r}\left(a_{l m, \text { ext }}(r)-\mu_{0} j_{l m}(r)\right)+\frac{\partial}{\partial r}\left(a_{l m, \text { ext }}(r)-\mu_{0} j_{l m}(r)\right) & =0 \\
\left(\frac{l(l+1)}{r^{2}}-\frac{2}{r} \frac{\partial}{\partial r}-\frac{\partial^{2}}{\partial r^{2}}\right) b_{l m, \text { ext }}(r) & =0 \\
\left(\frac{l}{r}-\frac{\partial}{\partial r}\right) a_{l m, \text { int }}(r) & =0 \\
\left(\frac{l+1}{r}+\frac{\partial}{\partial r}\right) a_{l m, \text { int }}(r) & =0 \\
\left(\frac{l(l+1)}{r^{2}}-\frac{2}{r} \frac{\partial}{\partial r}-\frac{\partial^{2}}{\partial r^{2}}\right) b_{l m, \text { int }}(r) & =0
\end{aligned}
$$

Together with the requirements that $\boldsymbol{B}_{l m}$ is finite at $r=0$ and when $r \rightarrow \infty$ and that $\boldsymbol{B}_{l m}$ is continuous at $r=R$, these equations give the expected result $a_{l m, \text { ext }}(r)=\mu_{0} j_{l m}(r)$ and $a_{l m, \text { int }}(r)=b_{l m, \text { int }}(r)=b_{l m, \text { ext }}(r)=0$. Thus, $\boldsymbol{B}_{l m}=0$ when $r<R$. This means that current densities of the form Eq. (B.11) do not contribute to the brain stimulation. Furthermore, because according to Eq. (A.12) $\boldsymbol{B}_{l m}$ at $r \geq R$ is orthogonal to the field produced by the shelltype current density, superposing these current densities to the shell-type current density would only increase the energy of the magnetic field (Section 2.2). Thus, we conclude that a single current-carrying layer as close to the spherically symmetric target volume as possible is the globally optimal solution for minimum-energy TMS coils. 


\section{References}

[1] Ruohonen J, Karhu J. Navigated transcranial magnetic stimulation. Clin Neurophysiol 2010;40:7-17.

[2] Deng Z-D, Lisanby SH, Peterchev AV. Electric field depth-focality tradeoff in transcranial magnetic stimulation: simulation comparison of 50 coil designs. Brain Stimul 2013;6:1-13.

[3] Barker AT, Jalinous R, Freeston IL. Non-invasive magnetic stimulation of human motor cortex. Lancet 1985;325:1106-7.

[4] Ueno S, Tashiro T, Harada K. Localized stimulation of neural tissues in the brain by means of a paired configuration of time-varying magnetic fields. J Appl Phys 1988;64:5862-4.

[5] Ren C, Tarjan PP, Popović DB. A novel electric design for electromagnetic stimulation - the slinky coil. IEEE Trans Biomed Eng 1995;42:918-25.

[6] Roth BJ, Maccabee PJ, Eberle LP, Amassian VE, Hallett M, Cadwell J, et al. In vitro evaluation of a 4-leaf coil design for magnetic stimulation of peripheral nerve. Electroen Clin Neuro 1994;93:68-74.

[7] Roth Y, Zangen A, Hallett M. A coil design for transcranial magnetic stimulation of deep brain regions. J Clin Neurophysiol 2002;19:361-70.

[8] Eaton H. Electric field induced in a spherical volume conductor from arbitrary coils: application to magnetic stimulation and MEG. Med Biol Eng Comput 1992;30:433-40.

[9] Sarvas J. Basic mathematical and electromagnetic concepts of the biomagnetic inverse problem. Phys Med Biol 1987;32:11-22.

[10] Jackson JD. Classical Electrodynamics. 3rd ed. New York: Wiley; 1999.

[11] Ilmoniemi RJ, Hämäläinen MS, Knuutila J. The forward and inverse problems in the spherical model. In: Weinberg H, Stroink G, Katila T, editors. Biomagnetism: Applications and Theory, New York: Pergamon Press; 1985, p. 278-82.

[12] Ilmoniemi RJ. The triangle phantom in magnetoencephalography. J Jpn Biomagn Bioelectromagn Soc 2009;22:44-5.

[13] Brasil-Neto JP, Cohen LG, Panizza M, Nilsson J, Roth BJ, Hallett M. Optimal focal transcranial magnetic activation of the human motor cortex: effects of coil orientation, shape of the induced current pulse, and stimulus intensity. J Clin Neurophysiol 1992;9:132-6.

[14] Ilmoniemi RJ, Ruohonen J, Karhu J. Transcranial magnetic stimulation - a new tool for functional imaging of the brain. Crit Rev Biomed Eng $1999 ; 27: 241-84$.

[15] Thielscher A, Kammer T. Electric field properties of two commercial figure8 coils in TMS: calculation of focality and efficiency. Clin Neurophysiol 2004;115:1697-708. 
[16] Komssi S, Kähkönen S, Ilmoniemi RJ. The effect of stimulus intensity on brain responses evoked by transcranial magnetic stimulation. Hum Brain Mapp 2004;21:154-64.

[17] Kähkönen S, Komssi S, Wilenius J, Ilmoniemi RJ. Prefrontal transcranial magnetic stimulation produces intensity-dependent EEG responses in humans. NeuroImage 2005;24:955-60.

[18] Boyd SP, Vandenberghe L. Convex Optimization. New York: Cambridge University Press; 2004.

[19] Peeren GN. Stream function approach for determining optimal surface currents. J Comput Phys 2003;191:305-21.

[20] Grover FW. Inductance Calculations, Working Formulas and Tables. New York: Van Nostrand Reinhold; 1946.

[21] Nieminen JO, Koponen LM, Ilmoniemi RJ. An automatic, computercontrolled TMS-coil calibrator. Clin Neurophysiol 2013;124:e163-4.

[22] Thielscher A, Kammer T. Linking physics with physiology in TMS: a sphere field model to determine the cortical stimulation site in TMS. NeuroImage $2002 ; 17: 1117-30$.

[23] Knäulein R, Weyh T. Minimization of energy stored in the magnetic field of air coils for medical application. 3rd International Workshop on Electric and Magnetic Fields 1996:477-82.

[24] Hsu KH, Durand DM. A 3-D differential coil design for localized magnetic stimulation. IEEE Trans Biomed Eng 2001;48:1162-8.

[25] Deng ZD, Lisanby SH, Peterchev AV. Coil design considerations for deep transcranial magnetic stimulation. Clin Neurophysiol 2014;125:1202-12.

[26] Epstein CM, Davey KR. Iron-core coils for transcranial magnetic stimulation. J Clin Neurophysiol 2002;19:376-81.

[27] Plonsey R, Heppner DB. Considerations of quasi-stationarity in electrophysiological systems. B Math Biophys 1967;29:657-64.

[28] Winch DE, Ivers DJ, Turner JPR, Stening RJ. Geomagnetism and Schmidt quasi-normalization. Geophys J Int 2005;160:487-504.

[29] Blatt JM, Weisskopf VF. Appendix B. Theoretical Nuclear Physics. New York: Springer-Verlag; 1952.

[30] Hill EL. The theory of vector spherical harmonics. Am J Phys 1954;22:21114.

[31] Stanaway SK, Cantwell BJ, Spalart PR. Appendix B. A numerical study of viscous vortex rings using a spectral method. Technical Memorandum 101041, NASA; 1988. 